\title{
Despersonalización en una muestra comunitaria y en personas con trastornos de ansiedad: el rol de las interpretaciones catastróficas y de la evitación experiencial
}

Rafael Kichic ${ }^{1}$

\section{Artículo}

Material original autorizado para la publicación en la revista Psicodebate. Facultad de Ciencias Sociales. Universidad de Palermo.

Recibido 18-06-2015 | Aceptado 14-08-2015

\section{Resumen}

Este estudio exploró la relación entre interpretaciones catastróficas, evitación experiencial y los diferentes subtipos de despersonalización (DP) en 70 participantes de la comunidad y 70 pacientes con trastornos de ansiedad. Los pacientes completaron instrumentos que medían interpretaciones catastróficas, evitación experiencial y DP. Las interpretaciones catastróficas predijeron la intensidad global de la DP y los subtipos experiencias corporales anómalas, experiencias anómalas del recordar, y desrealización. Contrariamente, la evitación experiencial no apareció como un predictor de DP. Los resultados son discutidos a la luz de los últimos avances en la conceptualización de la DP.

Palabras Clave: despersonalización, trastornos de ansiedad, interpretaciones catastróficas, evitación experiencial. 


\section{Abstract}

This study explored the relación among catastrophic interpretations, experiential avoidance and different subtypes of depersonalization (DP) in 70 individuals of the comunity and 70 individuals with anxiety disorders. Patients answered questionnaires that measured catastropihc interpretations, experiential avoidance and DP. Catastrophic interpretations predicted the global intensity of DP and also the subtypes anomalous corporal experiences, anomalous recall experiencies and derealization. On the opposite, experiential avoidance was not a predictor of DP. Results are commented in light of the progress in the conceptualization of DP.

Keywords: depersonalization, anxiety disorders, catastrophic interpretations, experiential avoidance. 
Los síntomas de despersonalización (DP) pueden configurar un trastorno en sí mismo, presentarse en el contexto de enfermedades neurológicas, en los diferentes trastornos de ansiedad (Barlow et al., 1985; Goff, Olin, Jenike, Baer \& Buttolph, 1992; Harvey \& Bryant, 1999; Hoyer, Braeuer, Crawcour, Klumbies \& Kirschbaum 2013; Litz \& Gray, 2002; Noyes et al., 1992), o bien estar presentes en la población general (Hunter, Sierra \& David, 2004). Por lo tanto, los participantes de la comunidad y los pacientes con trastornos de ansiedad constituyen grupos ideales para el estudio de la DP dado que estos síntomas son frecuentes en ambos grupos (Hunter et al., 2004) y se distribuyen a lo largo de un continuum de intensidad (Holmes et al., 2005).

A pesar de la existencia de modelos inscriptos en la tradición cognitivoconductual que intentan explicar la aparición y persistencia de los síntomas de DP (Hunter, Phillips, Chalder, Sierra \& David, 2003; Neziroglu \& Donnelly, 2010), poco se sabe acerca de variables psicológicas asociadas a estos síntomas. Desde el punto de vista clínico, explorar dicha asociación resulta importante para el diseño de estrategias terapéuticas, especialmente si se tiene en cuenta que la DP provoca un malestar intenso (Cox \& Swinson, 2002; Foa, Hembree \& Rothbaum 2007) y puede dificultar, en algunos casos, el tratamiento del trastorno ansioso (Foa et al., 2007; Jaycox \& Foa 1999; Lickel, Nelson, Hayes \& Deacon, 2008). Por lo tanto, un buen punto de partida son aquellos modelos que favorecieron el desarrollo de estrategias psicológicas eficaces para los trastornos de ansiedad (Hunter et al., 2003).

Basándose en estudios que indican que la DP es un síntoma frecuente en la población general, Hunter et al. (2004) propuso que las interpretaciones catastróficas de los síntomas de irrealidad provocan síntomas de ansiedad, lo cual motiva la evitación de situaciones disparadoras de DP. De esta manera, la DP es en sí misma objeto de interpretaciones disfuncionales como, por ejemplo, miedo a perder el control (Baker et al., 2007). De este modo, se esperaría que todas las personas con elevados niveles de DP presenten elevados niveles de ansiedad. Contrariamente, observaciones clínicas y hallazgos recientes sugieren que las personas con trastorno de DP pueden subdividirse en aquellas que informan altos y bajos niveles de ansiedad (Sierra, Medford, Wyatt, \& David 2012; Simeon \& Abugel, 2008). Esto representa un desafío para aquellos modelos que postulan una relación causal entre DP y ansiedad (Sierra et al., 2012).

Diferentes grupos de investigadores señalan que la percepción de pérdida de control subyace a las experiencias de DP (Foa, Riggs, \& Gershuny, 1995; Noyes \& Kletti, 1977; Sierra \& Berrios, 1998; Sierra \& David, 2007), por lo tanto, es posible que la percepción de peligro medie la relación entre ansiedad extrema y DP. Más específicamente, Sierra y Berrios (1998) postularon que la DP es una respuesta que permite lidiar con aquellas situaciones en donde la persona percibe 
que su vida está en peligro y no puede tomar distancia física respecto de la fuente de amenaza, no puede localizarla espacialmente, o resulta desconocida, es decir, la persona percibe que no tiene control. De este modo, no sólo la DP puede resultar un disparador de interpretaciones catastróficas sino también la percepción de pérdida de control en contextos ansiógenos también puede facilitar la aparición de la DP, como un equivalente funcional de las conductas de evitación, cuando luchar o huir no es posible (por ej., traumas prolongados, ataques de pánico).

Respecto del modelo de aceptación y compromiso, la evitación de experiencias negativas es propuesta como una variable asociada a la psicopatología en general (Hayes, Wilson, Gifford, Follete, Strosahl, 1996), y más recientemente a la DP (Neziroglu \& Donnelly, 2010). Sin embargo, esta relación no ha sido estudiada en pacientes con trastornos de DP u otros trastornos en los cuales dichos síntomas son frecuentes - por ej., los trastornos de ansiedad -.

La riqueza de las descripciones psicopatológicas clásicas ha impulsado la curiosidad por investigar si la variedad sintomática a la cual se refiere el término DP refleja una unidad subyacente que afecta los distintos aspectos de la experiencia (Sierra, Baker, Medford, \& David, 2005). Con el objetivo de capturar esta riqueza clínica y variedad sintomática, Sierra y Berrios (2000) desarrollaron la Cambridge Depersonalization Scale, un instrumento que mide las distintas variantes de la DP (Sierra, et al., 2005; Simeon \& Abugel, 2008).

Dado que un enfoque dimensional de la DP permite dilucidar en forma más adecuada los procesos cognitivos subyacentes al ampliar el rango de puntajes de las variables predictoras y la capacidad para predecir puntajes extremos (Graves, 2000), el presente estudio tiene por objetivo explorar la asociación entre la DP y variables derivadas del modelo cognitivo-conductual -interpretaciones catastróficas- y del modelo de la terapia de aceptación y compromiso (Hayes et al., 1996; Neziroglu \& Donnelly, 2010) - evitación experiencial -, en una muestra de participantes de la comunidad y de pacientes con diagnóstico de trastorno de ansiedad.

Nuestra hipótesis es que las interpretaciones catastróficas sobre los síntomas de ansiedad y la evitación experiencial están asociadas a la intensidad de la DP y sus diferentes subtipos en una muestra de pacientes con diagnóstico principal de trastornos de ansiedad y de participantes de la comunidad.

\section{Método}

\section{Muestra}

Para la muestra de participantes de la comunidad, se tomó una muestra intencional no probabilística de 70 participantes voluntarios adultos (49 mujeres; edad promedio $=33.5$ años, $\mathrm{DS}=12.1$, rango $=19-66)$ de la Ciudad Autónoma 
de Buenos Aires y el conurbano bonaerense durante el año 2012-2013. Los participantes no recibieron retribución económica por su participación en el estudio. Los criterios de inclusión eran: tener entre 18 y 70 años, ser de nacionalidad argentina, y ser capaz de completar los cuestionarios autoadministrables. Los criterios de exclusión fueron: haber sido diagnosticado previamente con un trastorno de ansiedad, síntomas psicóticos y/o epilepsia del lóbulo temporal.

La muestra de pacientes con trastornos de ansiedad estuvo compuesta por 70 adultos (41 mujeres; edad promedio $=36.2$ años, $\mathrm{DS}=11.9$, rango $18-66)$ con diagnóstico principal de trastorno de ansiedad que consultaron a la Clínica de Ansiedad y Estrés del Instituto de Neurología Cognitiva (INECO) de la Ciudad Autónoma de Buenos Aires en busca de tratamiento durante los años 2012 y 2013. La muestra fue de tipo intencional no probabilística. Cada participante completó una batería de escalas autoadministrables que forman parte de la evaluación de rutina de la Clínica de Ansiedad y Estrés. El diagnóstico de los pacientes fue realizado por un experto en trastornos de ansiedad. Los criterios de inclusión eran: tener un trastorno de ansiedad como diagnóstico principal, nacionalidad argentina, y ser capaz de completar los cuestionarios autoadministrables. Los criterios de exclusión fueron: ser menor de 18 años, presencia de síntomas psicóticos, imposibilidad de completar los cuestionarios, retraso mental y epilepsia del lóbulo temporal. Los participantes cumplieron criterios para los siguientes diagnósticos del DSM-IV (APA, 1994): trastorno obsesivo-compulsivo (40\%), trastorno de pánico con o sin agorafobia (24.3\%), agorafobia sin historia de trastorno de pánico $(4.3 \%)$, trastorno de ansiedad generalizada (11.4\%), trastorno por estrés postraumático $(8.6 \%)$, fobia social $(5.7 \%)$, y fobia específica $(5.7 \%)$.

\section{Instrumentos}

Datos demográficos: se diseñó un cuestionario autoadministrable que pregunta sobre la edad, género, y nacionalidad del participante.

Structured Clinical Interview for DSM-IV Axis I Disorders, Research Version, Patient Edition With Psychotic Screen, Bilingual Edition (SCID-I/P W/PSY SCREEN; First, Spitzer, Gibbon, \& Williams, 1998). Módulo F. Para establecer el diagnóstico principal de trastorno de ansiedad en la muestra clínica se empleó el módulo de trastornos de ansiedad - módulo F - de la SCID-I. La SCID-I sigue los criterios diagnósticos especificados en el DSM-IV (American Psychiatric Association, 1994; First, Gibbon, Spitzer, \& Williams, 1996).

Presencia o ausencia de trastornos de ansiedad, epilepsia del lóbulo temporal, y sintomas psicóticos: se diseñó un cuestionario autoadministrable con el objetivo de evaluar la presencia o ausencia de un trastorno de ansiedad, síntomas psicóticos o epilepsia del lóbulo temporal en los participantes de la comunidad. 
Este cuestionario tiene un formato de respuesta de SI-NO y consiste en 8 ítems que preguntan si la persona ha sido diagnosticada con alguno de los trastornos de ansiedad reconocidos en el DSM-IV (American Psychiatric Association, 1994).

Cambridge Depersonalization Scale (CDS; Sierra \& Berrios, 2000): es un cuestionario autoadministrable de 29 ítems que permiten evaluar la intensidad de los síntomas de DP en los últimos 6 meses. El puntaje total está compuesto por la suma de todos los ítems. Estudios de análisis factorial arrojaron una solución de cuatro factores (Sierra et al., 2005). Estos fueron: a) experiencias corporales anómalas (ECA), b) embotamiento afectivo (EA), c) experiencias anómalas del recordar (EAR), y d) desrealización (DR). En este trabajo se empleó la versión española desarrollada por Molina Castillo et al. (2006).

Agoraphobic Cognitions Questionnaire (ACQ; Chambless, Caputo, Bright \& Gallagher, 1984): este cuestionario autoadministrable evalúa interpretaciones catastróficas asociadas a la presencia de síntomas de ansiedad. Está compuesto de 14 ítems que se puntúan de 1 (ese pensamiento nunca ocurre) a 5 (ese pensamiento ocurre siempre). El ACQ comprende dos subescalas: interpretaciones o creencias catastróficas acerca de la pérdida de control (PC) - por ej., "voy a gritar" - e interpretaciones o creencias catastróficas acerca de padecer enfermedades físicas potencialmente mortales (PF) -por ej., "voy a tener un ataque al corazón"-.

Body Sensations Questionnaire (BSQ; Chambless et al., 1984): este cuestionario evalúa la preocupación por la presencia de síntomas físicos en una escala Likert de 1 (esta sensación no me preocupa para nada) a 5 (esta sensación me preocupa extremadamente). El puntaje total está compuesto por la suma de todos los ítems.

Acceptance and Action Questionnaire (AAQ-9: Hayes, 2004): este instrumento autoadministrable evalúa la evitación de experiencias internas negativas (pensamientos, síntomas físicos, emociones, etc.) o evitación experiencial. Está compuesto por 9 ítems que se puntúan de 1 (nunca es cierto) a 7 (siempre es cierto).

Beck Depression Inventory-II (BDI-II; Beck, Steer \& Brown, 1996): es un instrumento autoadministrable que mide la severidad de los síntomas depresivos.

State Trait Anxiety Inventory- Trate Scale (STAI-T; Spielberger, Gorsuch, Lushene, Vagg \& Jacobs, 1983): este instrumento autoadministrable es ampliamente usado para medir ansiedad en general. La subescala rasgo de la STAI está compuesta por 20 ítems y pregunta a los sujetos por la frecuencia con la cual generalmente presentan sentimientos, cogniciones y síntomas relacionados con la ansiedad.

\section{Procedimiento}

Los participantes de la comunidad fueron reclutados mediante conocidos del investigador. Antes de comenzar a completar las medidas autoadministrables, se 
pidió un consentimiento informado a cada participante en el cual se explicaba el tipo de investigación y se solicitaba su autorización voluntaria para participar en la misma. Los participantes debían responder preguntas acerca de si fue diagnosticado con un trastorno de ansiedad, con síntomas psicóticos o epilepsia del lóbulo temporal. Luego los participantes de la comunidad completaron preguntas sobre variables demográficas y las siguientes medidas: CDS, ACQ, BSQ, BDI-II, STAI-T, OCI-R, III-31, AAQ.

La muestra de pacientes con diagnóstico principal de trastorno de ansiedad estuvo constituida por aquellos pacientes que consultaron en busca de tratamiento a la Clínica de Ansiedad y Estrés del Instituto de Neurología Cognitiva (INECO) de la Ciudad Autónoma de Buenos Aires. Antes de comenzar a completar las medidas autoadministrables, se pidió un consentimiento informado a cada paciente, en el cual se explicaba el tipo de investigación y se solicitaba de su autorización voluntaria para participar en la misma. Los pacientes completaron un paquete de medidas autoadministrables que incluían: variables demográficas, CDS, ACQ, BSQ, BDI-II, STAI-T, OCI-R, III-31, AAQ. Estas medidas eran completadas antes de realizar la/s entrevista/s de evaluación en la Clínica de Ansiedad y Estrés de INECO o bien entre entrevistas de evaluación. Durante la o las entrevistas se aplicó la entrevista semi-estructurada SCID-I módulo F. Las entrevistas de evaluación fueron realizadas por un psicólogo experto en el diagnóstico y tratamiento de los trastornos de ansiedad. El estudio fue aprobado por el comité de ética de INECO.

\section{Resultados}

La muestra completa $(n=140)$ presentó los siguientes puntajes promedio en los instrumentos administrables: CDS puntaje total = 21.4 (DS 24.8); ACQ puntaje total $=1.6(D S .58)$; ACQ-subescala creencias de pérdida de control $(\mathrm{ACQ}-\mathrm{PC})=1.8(D S .73)$; ACQ-subescala preocupaciones físicas $(\mathrm{ACQ}-\mathrm{PF})=1.5$ $(D S .62) ; \mathrm{AAQ}=35.8(D S$ 8.1); $\mathrm{STAI}-\mathrm{T}=25.8(D S 13.5) ; \mathrm{BSQ}=30.5(D S 12.2)$; $\mathrm{BDI}-\mathrm{II}=14.4(D S 12.5)$.

\section{Análisis de regresión}

A fin de examinar la asociación entre las interpretaciones catastróficas, la evitación experiencial y la DP, se realizó una serie de análisis de regresión para determinar si estas variables, la depresión, la ansiedad, y la preocupación por los síntomas del pánico predecían los síntomas de DP y cada uno de sus subtipos. Todos los análisis de regresión fueron analizados sobre la muestra de participantes de la comunidad y la muestra de pacientes con trastornos de ansiedad en forma conjunta. 
Dado que las correlaciones entre el puntaje total de la ACQ con la ACQ-PF $(.83, \mathrm{p}=.000)$, ACQ-PC $(.89, \mathrm{p}=.000)$ y BSQ $(.75, \mathrm{p}=.000)$ fueron altas y significativas, se decidió excluir del análisis de regresión el puntaje total de la ACQ para disminuir el riesgo de multicolinelidad. En todos los modelos finales de regresión el valor de la tolerancia fue mayor a 0.10 y los factores de inflación de la varianza fueron menores a 10 , lo cual permite descartar la posible presencia de multicolinealidad entre las variables independientes (Cohen et al., 2003).

Para determinar la presencia de casos atípicos univariados en la variable dependiente se examinaron los residuos estudientizados. Si el valor del residuo era \pm 3 entonces se consideraba que había un caso atípico (Stevens, 1984). Para determinar la presencia de casos atípicos multivariados en las variables independientes se calcularon los puntajes de distancia de Mahalanobis $\left(\mathrm{D}^{2}\right)$ (Stevens, 1984). Si la probabilidad de $\mathrm{D}^{2}$ era $p<.001$ se consideraba que había un caso atípico. Cuando se detectaron casos atípicos univariados o multivariados se recalcularon las ecuaciones de regresión lineal excluyendo dichos casos. Los coeficientes de regresión informados en las tablas corresponden a los análisis de regresión en donde se excluyen los casos atípicos.

Siguiendo estos criterios se calculó la regresión lineal con el puntaje global transformado de la CDS como variable dependiente. El $\mathrm{R}^{2}$ ajustado indica que el $64.3 \%$ de la variabilidad de la intensidad global de la DP fue explicada por la depresión, las interpretaciones catastróficas y la ansiedad.

Cuando se calculó la regresión lineal con el puntaje transformado de la subescala de experiencias corporales anómalas de la CDS (CDS-ECA) como variable dependiente, se obtuvo un $\mathrm{R}^{2}$ ajustado .413 . El $\mathrm{R}^{2}$ ajustado obtenido sin casos atípicos fue .469 . El valor de $\mathrm{R}^{2}$ ajustado sin casos atípicos indica que el 46.9 $\%$ de la variabilidad de las experiencias corporales anómalas fue explicada por la depresión y las interpretaciones catastróficas acerca de padecer enfermedades.

Al momento de realizar la regresión lineal con el puntaje transformado de la subescala de embotamiento afectivo de la CDS (CDS-EA) como variable dependiente, se obtuvo un $\mathrm{R}^{2}$ ajustado de .459 . El $\mathrm{R}^{2}$ ajustado obtenido sin caso atípico fue .461 . El valor de $\mathrm{R}^{2}$ ajustado sin casos atípicos indica que el $46.1 \%$ de la variabilidad de la intensidad del embotamiento afectivo fue explicada por la depresión y el malestar asociado a los síntomas del pánico.

Cuando se ingresó el puntaje transformado de la subescala experiencias anómalas del recordar de la CDS (CDS-EAR) como variable dependiente, el $\mathrm{R}^{2}$ ajustado obtenido fue .224 . El $\mathrm{R}^{2}$ ajustado obtenido sin casos atípicos fue .228 , indicando que el $22.8 \%$ de la variabilidad de la intensidad de las experiencias anómalas del recordar fue explicada por la ansiedad y las interpretaciones catastróficas acerca de la pérdida de control. 
Por último, se ingresó el puntaje transformado de la subescala de desrealización de la CDS (CDS-DR) como variable dependiente. El $\mathrm{R}^{2}$ ajustado obtenido fue .606 , mientras que al excluir los casos atípicos fue de .617. El valor de $\mathrm{R}^{2}$ ajustado sin casos atípicos indica que la depresión y las interpretaciones catastróficas acerca de padecer enfermedades explicaron el $61.7 \%$ de la variabilidad de la intensidad de la desrealización.

La Tabla 1 muestra un resumen de aquellas variables independientes retenidas por el procedimiento para la predicción de la intensidad de la DP y sus diferentes subtipos.

\section{Tabla 1.}

Resumen de los Resultados de Todas las Regresiones Lineales Múltiples con el Método de Selección de Variables por Pasos en Donde se Emplean la ACQ, BSQ, AAQ, STAI-T, y BDI-Il para Predecir el Puntaje Global Transformado de la CDS y cada una de sus Subescalas en la Muestra de Pacientes con Trastornos de Ansiedad y Participantes de la Comunidad

\begin{tabular}{lcc}
\hline Variable dependiente & Variable independiente & $\boldsymbol{R}^{2}$ ajustado \\
\hline CDS-Puntaje total & BDI-II, ACQ-PC, ACQ-PF, STAI-T & .643 \\
\hline Experiencias corporales anómalas & BDI-II, ACQ-PF & .469 \\
\hline Embotamiento afectivo & BDI-II, BSQ & .461 \\
\hline Experiencias anómalas del recordar & STAI-T, ACQ-PC & .228 \\
\hline Desrealización & BDI-II, ACQ-PF & .617 \\
\hline
\end{tabular}

Nota. CDS = Cambridge Depersonalization Scale; $A C Q=$ Agoraphobic Cognitions Questionnaire; $\mathrm{PC}=$ pérdida de control; $\mathrm{PF}=$ preocupaciones físicas; $\mathrm{AAQ}=$ Acceptance and Action Questionnaire; STAI-T = State Trait Anxiety Inventory-Trait Scale; BSQ = Body Sensations Questionnaire; BDI-II = Beck Depression Inventory-II.

\section{Discusión}

La distribución continua que caracteriza a la DP (Holmes et al., 2005) nos llevó a explorar los procesos cognitivos subyacentes a la DP en las muestras de participantes de la comunidad y de pacientes con trastornos de ansiedad en forma conjunta.

De acuerdo con nuestra hipótesis, las interpretaciones catastróficas predijeron la intensidad global de la DP y los subtipos experiencias corporales anómalas, experiencias anómalas del recordar, y desrealización. Más específicamente, la percepción de pérdida de control predijo los síntomas de experiencias anómalas del recordar, y las creencias acerca de padecer enfermedades físicas predijeron la intensidad de las experiencias corporales anómalas y de la desrealización. 
Podría especularse que la estrecha relación entre el ensimismamiento y las anomalías subjetivas del recordar (Sierra et al., 2005) supone que este subtipo comprende síntomas parecidos a los experimentados durante un trance. En ese sentido, el síntoma de sensación de vacío mental ("mind emptiness"), que forma parte de este subtipo, ha sido considerado por Sierra y Berrios (1998) como el equivalente mental de la inmovilidad conductual ("freezing") en situaciones en donde la persona percibe que luchar o huir frente a un peligro no es posible, dado que se presenta en estados de activación autonómica intensos. De este modo, podría explicarse la relación encontrada entre las creencias de pérdida de control y las anomalías subjetivas del recordar.

Respecto de la relación entre los temores a una enfermedad física y las experiencias corporales anómalas, es posible que aquellos que sufren la experiencia de no habitar su propio cuerpo presenten preocupaciones más intensas por estar padeciendo una enfermedad física mortal e inminente - por ej., una enfermedad neurológica -. Esta explicación es congruente con la atribución causal a un daño cerebral que suelen realizar los pacientes con DP crónica (Baker et al., 2007; Hunter, Salkovskis, \& David, 2014) y los postulados teóricos del modelo de Hunter et al. (2003).

En su conjunto, nuestros resultados apoyan parcialmente nuestra hipótesis según la cual la intensidad de la DP y sus diferentes subtipos está asociada a las interpretaciones catastróficas de los síntomas de ansiedad. Además, nuestros resultados son consistentes con el modelo cognitivo-conductual de la DP (Hunter et al., 2003) y con la modificación propuesta para este modelo según la cual las interpretaciones catastróficas subyacen al desarrollo y mantenimiento de la DP.

En contraposición a nuestra hipótesis, la evitación experiencial no apareció como predictor de la DP. En consecuencia, nuestros resultados sugieren que la evitación experiencial no parece jugar un rol importante en el desarrollo y mantenimiento de la DP de personas con trastornos de ansiedad o de la comunidad. Diferencias metodológicas podrían explicar los resultados contradictorios entre los obtenidos en este estudio y el de Michal et al. (2007). A diferencia del estudio de Michal et al., en donde una medida de atención plena estuvo asociada con la DP y predijo su intensidad en pacientes con dolor y controles, en nuestro estudio se incluyeron pacientes ansiosos y se empleó una medida indirecta de aceptación, constructo que se superpone parcialmente con el constructo de atención plena (Block-Lerner, Salters-Pedneault \& Tull, 2005).

El fracaso de la evitación experiencial para explicar un porcentaje adicional de la varianza de la DP es congruente con las dificultades de la evitación experiencial en predecir sintomatología ansiosa en personas con trastornos de ansiedad (Berman, Wheaton, McGrath \& Abramowitz, 2010; Manos et al., 2010) y en muestras no clínicas (Abramowitz, Lackey \& Wheaton, 2009; Mahaffey, 
Wheaton, Fabricant, Berman, \& Abramowitz, 2013). En general, en estos estudios las creencias específicas asociadas a la ansiedad o alguno de sus trastornos fueron mejores predictores de la sintomatología ansiosa que la evitación experiencial, medida esta última con diferentes versiones de la AAQ. Como lo han sugerido Abramowitz et al. (2009) y Manos et al. (2010), la evitación experiencial, medida por la $\mathrm{AAQ}$, refleja una tendencia global a evitar eventos internos, mientras que las creencias propias de cada cuadro diagnóstico o bien las interpretaciones catastróficas sobre la ansiedad en nuestro caso, reflejan un constructo más específico para los trastornos de ansiedad. Por lo tanto, medidas de evitación experiencial que resulten específicas para determinados trastornos, como la DP, quizás resulten más apropiadas para estudiar su relación con los diferentes síndromes (Manos et al., 2010).

En forma consistente con estudios previos (Baker et al., 2003; Baker et al., 2007; Cox \& Swinson, 2002; Michal et al., 2011; Trueman, 1984a, 1984b), la depresión y la ansiedad aparecieron como predictores de la intensidad global de la DP. A diferencia de la ansiedad, la depresión predijo tres de los cuatro subtipos de DP, lo cual indica la relevancia de los síntomas depresivos para el estudio psicopatológico de la DP.

Este estudio expande los conocimientos preexistentes en el área de la DP en población ansiosa y comunitaria de forma significativa al investigar postulados teóricos del modelo cognitivo-conductual (Hunter et al., 2003) y de aceptación y compromiso (Hayes et al., 1996; Neziroglu \& Donnelly, 2010). Según nuestro conocimiento, este es el primer estudio en brindar apoyo empírico e investigar sistemáticamente la relación entre creencias catastróficas, evitación experiencial, y los diferentes subtipos de DP.

Los resultados expuestos deben ser interpretados con precaución debido a las limitaciones de este estudio. En primer lugar, el diseño transversal no permite sacar conclusiones acerca de la relación causal entre las variables estudiadas y la DP. De este modo, estudios que manipulen experimentalmente las variables teóricas, como creencias acerca del control, pueden arrojar luz acerca de la relación causal entre las interpretaciones catastróficas de la ansiedad y la DP (Sanderson, Rapee, \& Barlow 1989). En esta línea, los resultados de las tareas experimentales empleadas por Hunter et al. (2014) brindan un apoyo inicial a la hipótesis de una asociación entre interpretaciones catastróficas sobre la DP y el mantenimiento de la misma.

En segundo lugar, para descartar la presencia de un trastorno de ansiedad u otros síntomas neuropsiquiátricos en la muestra de participantes de la comunidad se emplearon una serie de preguntas autoadministrables, en lugar de una entrevista semi-estructurada, lo cual hubiera permitido un mayor grado de certeza acerca de 
la ausencia de patología. En tercer lugar, al no haberse evaluado la confiabilidad entre evaluadores para la administración de la SCID-I/P se podría cuestionar la fiabilidad de los diagnósticos establecidos para la muestra clínica. No obstante, es importante subrayar que el evaluador tenía amplia experiencia en el diagnóstico de los trastornos de ansiedad.

Por último, este trabajo fue realizado antes de la publicación del DSM-5 y posiblemente nuestras conclusiones acerca de la relación entre las variables estudiadas y la DP no puedan generalizarse a la nueva clasificación. De cualquier forma, los criterios sintomáticos de las crisis de angustia y de los trastornos de ansiedad tal como se conciben en el DSM-IV no sufrieron cambios sustanciales.

Tomando en cuenta estas limitaciones, los resultados obtenidos sugieren que las interpretaciones catastróficas de los síntomas de ansiedad parecen reflejar en una forma más adecuada los procesos cognitivos subyacentes a la DP que el constructo de la evitación experiencial, y por lo tanto deberían ser uno de los objetivos clínicos centrales para reducir la DP.

\section{Referencias}

Abramowitz, J. S., Lackey, G. R., \& Wheaton, M. G. (2009). Obsessivecompulsive symptoms: The contribution of obsessional beliefs and experiential avoidance. Journal of Anxiety Disorders, 23(2), 160-166.

American Psychiatric Association (1994). Diagnostic and statistical manual of mental disorders (4th ed.). Washington, D.C.: Author.

Baker, D., Earle, M., Medford, N., Sierra, M., Towell, A., \& David, A. (2007). Illness perceptions in depersonalization disorder: testing an illness attribution model. Clinical Psychology \& Psychotherapy, 14(2), 105-116.

Baker, D., Hunter, E., Lawrence, E., Medford, N., Patel, M., Senior., \& David, A. S. (2003). Depersonalisation disorder: clinical features of 204 cases. The British Journal of Psychiatry, 182(5), 428-433.

Barlow, D. H., Vermilyea, J., Blanchard, E. B., Vermilyea, B. B., Di Nardo, P. A., \& Cerny, J. A. (1985). The phenomenon of panic. Journal of Abnormal Psychology, 94(3), 320.

Beck, A. T., Steer, R. A., \& Brown, G. K. (1996). Beck Depression InventoryManual. San Antonio, TX: The Psychological Corporation. 
Berman, N. C., Wheaton, M. G., McGrath, P., \& Abramowitz, J. S. (2010). Predicting anxiety: The role of experiential avoidance and anxiety sensitivity. Journal of Anxiety Disorders, 24(1), 109-113.

Block-Lerner, J., Salters-Pedneault, K., \& Tull, M. T. (2005). Assessing mindfulness and experiential acceptance. En S. M. Orsillo \& L. Roemer (Eds). Acceptance and mindfulness-based approaches to anxiety (pp. 71-99). Springer US.

Chambless, D. L., Caputo, G. C., Bright, P., \& Gallagher, R. (1984). Assessment of fear of fear in agoraphobics: the body sensations questionnaire and the agoraphobic cognitions questionnaire. Journal of consulting and clinical psychology, 52(6), 1090.

Cohen, J., Cohen, P., \& Stephen, G. West, and Leona S. Aiken. (2003). Applied multiple regression/correlation analysis for the behavioral sciences ( $3 \mathrm{rd}$ ed.). Mahwah: Erlbaum.

Cox, B. J., \& Swinson, R. P. (2002). Instrument to assess depersonalization $\square$ derealization in panic disorder. Depression and anxiety, 15(4), 172-175.

First, M. B., Spitzer, R. L., Gibbon, M., \& Williams, J. B. W. (1996). User's guide for the Structured Clinical Interview for DSM-IV Axis I Disorders, Research Version. New York: Biometrics Research, New York State Psychiatric Institute.

First, M. B., Spitzer, R. L., Gibbon, M. \& Williams, J. B. W. (1998). Structured Clinical Interview for DSM-IV Axis I Disorders, Research Version, Patient Edition with Psychotic Screen (SCID-I/P W/PSY SCREEN). New York: Biometrics Research, New York State Psychiatric Institute.

Foa, E. B., Hembree, E. A., \& Rothbaum, B. O. (2007). Prolonged exposure therapy for PTSD: Emotional processing of traumatic experiences. Oxford: Oxford University Press.

Foa, E. B., Riggs, D., \& Gershuny, B. (1995). Arousal, numbing, and intrusion. American Journal of Psychiatry, 152, 116-120.

Goff, D. C., Olin, J. A., Jenike, M. A., Baer, L., \& Buttolph, M. L. (1992). Dissociative symptoms in patients with obsessive-compulsive disorder. The Journal of nervous and mental disease, 180(5), 332-337.

Graves, R. E. (2000). Accuracy of regression equation prediction across the range of estimated premorbid IQ. Journal of clinical and experimental neuropsychology, 22(3), 316-324. 
Harvey, A. G., \& Bryant, R. A. (1999). Dissociative symptoms in acute stress disorder. Journal of traumatic stress, 12(4), 673-680.

Hayes, S. C. (2004). Acceptance and Commitment Therapy and the New Behavior Therapies: Mindfulness, Acceptance, and Relationship. En S. C. Hayes, V. M. Follete, \& M. Linehan (Eds.), Mindfulness and acceptance: Expanding the cognitive behavioral tradition (pp. 1-29). New York: Guilford.

Hayes, S. C., Wilson, K. G., Gifford, E. V., Follette, V. M., \& Strosahl, K. (1996). Experiential avoidance and behavioral disorders: a functional dimensional approach to diagnosis and treatment. Journal of consulting and clinical psychology, 64(6), 1152.

Holmes, E. A., Brown, R. J., Mansell, W., Fearon, R. P., Hunter, E. C., Frasquilho, F., \& Oakley, D. A. (2005). Are there two qualitatively distinct forms of dissociation? A review and some clinical implications. Clinical psychology review, 25(1), 1-23.

Hoyer, J., Braeuer, D., Crawcour, S., Klumbies, E., \& Kirschbaum, C. (2013). Depersonalization/derealization during acute social st ress in social phobia. Journal of anxiety disorders, 27(2), 178-187.

Hunter, E. C. M., Phillips, M. L., Chalder, T., Sierra, M., \& David, A. S. (2003). Depersonalisation disorder: a cognitive-behavioural conceptualisation. Behaviour Research and Therapy, 41(12), 1451-1467.

Hunter, E. C., Salkovskis, P. M., \& David, A. S. (2014). Attributions, appraisals and attention for symptoms in depersonalisation disorder. Behaviour research and therapy, 53, 20-29.

Hunter, E. C., Sierra, M., \& David, A. S. (2004). The epidemiology of depersonalisation and derealisation. Social psychiatry and psychiatric epidemiology, 39(1), 9-18.

Jaycox, L. H., \& Foa, E. B. (1999). Obstacles in implementing exposure therapy for PTSD: Case discussions and practical solution. Clinical psychology and Psychotherapy, 3, 176-184.

Lickel, J., Nelson, E., Hayes, A., \& Deacon, B. (2008). Interoceptive exposure exercises for evoking depersonalization and derealization: A pilot study. Journal of Cognitive Psychotherapy, 22, 321-330.

Litz, B. T., Litz, B. T., \& Gray, M. J. (2002). Emotional numbing in posttraumatic 
stress disorder: Current and future research directions. Australian and New Zealand Journal of Psychiatry, 36(2), 198-204.

Mahaffey, B. L., Wheaton, M. G., Fabricant, L. E., Berman, N. C., \& Abramowitz, J. S. (2013). The contribution of experiential avoidance and social cognitions in the prediction of social anxiety. Behavioural and cognitive psychotherapy, 41(01), 52-65.

Manos, R. C., Cahill, S. P., Wetterneck, C. T., Conelea, C. A., Ross, A. R., \& Riemann, B. C. (2010). The impact of experiential avoidance and obsessive beliefs on obsessive-compulsive symptoms in a severe clinical sample. Journal of anxiety disorders, 24(7), 700-708.

Michal, M., Beutel, M. E., Jordan, J., Zimmermann, M., Wolters, S., \& Heidenreich, T. (2007). Depersonalization, mindfulness, and childhood trauma. The Journal of nervous and mental disease, 195(8), 693-696.

Michal, M., Glaesmer, H., Zwerenz, R., Knebel, A., Wiltink, J., Brähler, E., $\&$ Beutel, M. E. (2011). Base rates for depersonalization according to the 2-item version of the Cambridge Depersonalization Scale (CDS-2) and its associations with depression/anxiety in the general population. Journal of affective disorders, 128(1), 106-111.

Molina Castillo, F., Molina Castillo, J. J., Martínez de la Iglesia, J., Albert Colomer, C., Berrios, G., Luque Luque, R., \& Sierra, A. M. (2006). Adaptación y validación al castellano de la Escala de Despersonalización de Cambridge. Actas Españolas de Psiquiatría, 34(3), 185-192.

Neziroglu, F. \& Donnelly, K. (2010). Overcoming Depersonalization Disorder: A mindfulness and acceptance guide to conquering feelings of numbness and unreality. Oakland: New Harbinger Publications.

Noyes, R., \& Kletti, R. (1977). Depersonalization in response to life-threatening danger. Comprehensive psychiatry, 18(4), 375-384.

Noyes, R., Woodman, C., Garvey, M. J., Cook, B. L., Suelzer, M., Clancy, J., \& Anderson, D. J. (1992). Generalized anxiety disorder vs. panic disorder: Distinguishing characteristics and patterns of comorbidity. The Journal of nervous and mental disease, 180(6), 369-379.

Sanderson, W. C., Rapee, R. M., \& Barlow, D. H. (1989). The influence of an illusion of control on panic attacks induced via inhalation of $5.5 \%$ carbon 
dioxide-enriched air. Archives of General Psychiatry, 46(2), 157-162.

Sierra, M., Baker, D., Medford, N., \& David, A. S. (2005). Unpacking the depersonalization syndrome: an exploratory factor analysis on the Cambridge Depersonalization Scale. Psychological medicine, 35(10), 1523-1532.

Sierra, M., \& Berrios, G. E. (1998). Depersonalization: Neurobiological perspectives. Biological Psychiatry, 44, 898-908.

Sierra, M., \& Berrios, G. E. (2000). The Cambridge Depersonalisation Scale: a new instrument for the measurement of depersonalisation. Psychiatry research,93(2), 153-164.

Sierra, M., \& David, A. S. (2007). Depersonalization and individualism: the effect of culture on symptom profiles in panic disorder. The Journal of nervous and mental disease, 195(12), 989-995.

Sierra, M., Medford, N., Wyatt, G., \& David, A. S. (2012). Depersonalization disorder and anxiety: a special relationship?. Psychiatry research, 197(1), 123127.

Simeon, D. S. \& Abugel, J. (2008). Feeling unreal: Depersonalization disorder and the loss of the self. Oxford: Oxford University Press.

Spielberger, C. D., Gorsuch, R. L., Lushene, R. E., Vagg, P. R., \& Jacobs, G. A. (1983). Manual for the State-Trait Anxiety Inventory STAI. Mountain View, CA: Consulting Psychologists Press.

Stevens, J. P. (1984). Outliers and influential data points in regression analysis. Psychological Bulletin, 95, 334-344.

Trueman, D. (1984a). Depersonalization in a nonclinical population. The Journal of psychology, 116(1), 107-112.

Trueman, D. (1984b). Anxiety and depersonalization and derealization experiences. Psychological reports, 54(1), 91-96. 